\title{
Finite element modeling of K-Monel bolts under static loading and dynamic shock loading
}

\author{
Kevin Behan*, Emily Guzas*, Jeffrey Milburn and Stacy Moss \\ US Naval Undersea Warfare Center, Division Newport (NUWCDIVNPT), Newport, RI, USA
}

Received 2 February 2012

Revised 19 September 2012

Accepted 7 December 2012

\begin{abstract}
The Naval Undersea Warfare Center has funded a project to investigate the accuracy of various bolt models used to represent actual shipboard bolted connections within an analytical shock survivability assessment. The ultimate goal within this project is to develop finite element bolt representations that are not only computationally efficient, but also accurate. A significant task within this effort involved the development of highly detailed finite element models of bolted connections under various load configurations. Accordingly, high-resolution bolt models were developed and incorporated into simulations of four bolted connection test arrangements: static shear, static tension, dynamic shear, and dynamic tension. These simulation results are validated against experimental data from physical testing of each configuration. Future research will focus on exploring simplified finite element bolt representations and comparing these against the high-resolution results.
\end{abstract}

Keywords: Finite element analysis, bolted joints, K-Monel bolts, validation, static loading, shock loading

\section{Introduction}

The analytical assessment of shock survivability of naval systems and components typically involves developing complex, detailed finite element models of individual components, sub-assemblies, and the systems themselves. Components of these systems often include bolted connections, which are necessarily incorporated into the finite element models. Explicit modeling of bolts, say with continuum elements, is uncommon in standard practice because of model size and run time limitations: bolted connections are often modeled with rudimentary simplified bolt representations, such as a single, rigid element, or one or more beam elements. Thus, finite element-based shock survivability assessments become partially dependent on the accuracy of these bolt representations, and the use of such models to evaluate bolt survivability often leads to highly conservative results and overdesigned connections.

A first step towards improving the accuracy of simplified bolt representations involves calibration of numerical models of bolts to experimental data of bolted connections under a variety of loading configurations. To aid in this calibration, the current research effort focused on developing highly detailed finite element models of bolt arrangements as tested in actual physical experiments, and comparing simulation results against experimental data from the bolts tested under static and dynamic shear and tensile loading. The intent here is to provide a benchmark for future research on the evaluation of simplified finite element bolt representations.

${ }^{*}$ Corresponding author: Emily Guzas, Kevin Behan, US Naval Undersea Warfare Center, Division Newport, 1176 Howell Street, Newport, RI 02841, USA. Tel.: +1 401832 5577; Fax: +1 401832 7207; E-mail: emily.guzas@navy.mil, kevin.behan@navy.mil. 
Table 1

K500 physical properties (strength properties from tests by Thielsch Engineering, Inc. on 12 October 2010)

\begin{tabular}{ccccc}
\hline Density $\left(\mathrm{g} / \mathrm{cm}^{3}\right)$ & $\begin{array}{c}\text { Elastic modulus } \\
(\mathrm{GPa})\end{array}$ & Poisson's ratio & $\begin{array}{c}\text { Yield strength, } \\
0.2 \% \text { offset }(\mathrm{MPa})\end{array}$ & $\begin{array}{c}\text { Ultimate strength } \\
(\mathrm{MPa})\end{array}$ \\
\hline 8.435 & 178.2 & 0.32 & 729.5 & $\begin{array}{c}\text { Elongation in 4D } \\
(\%)\end{array}$ \\
\hline
\end{tabular}

Previous work regarding detailed finite element modeling of bolted connections with validation through experiment has focused a variety of joint types and loading configurations. McCarthy et al. presented results on single-lap, single-bolt composite joints with titanium bolts $[13,14]$. Base model development and validation against experimental strain gauge data from the joint surface and experimental joint stiffness data is discussed in [13], and [14] examines the effects of bolt clearance on various aspects of joint behavior including joint stiffness, bolt rotation, bolt-hole contact area, stress distribution in the laminate, and the onset of failure. Chung and Ip [8,9] evaluated the structural response of bolted connections between cold-formed steel strips and hot rolled steel plates for grade 8.8 bolts under shear loading. In [8], Chung and Ip presented results from shear experiments on G300 and G550 cold-formed steel specimens and outlined modeling techniques used to validate their numerical results against experimental load-deflection data. This research is extended in [9], where the validated models are used to investigate bearing failure, shear-out failure and net section failure in lap shear tests, and to perform parametric studies to examine the effects of different steel constitutive characteristics on connection bearing resistance.

Past work has also focused on simplified finite element modeling of bolted connections, including modeling of steel beam-column connections consisting of single plate, shear-tab connections that were fillet welded to steel columns and bolted to the shear tabs using high-strength bolts [11]. In particular, Khandelwal et al. [11,12] described the development and calibration of connection macromodels (combinations of beam and discrete spring elements) capable of capturing the dynamic response behavior of various steel building frame connections, where these macromodels were then used to examine the collapse resistance of planar ten-story steel moment frames. A similar approach as applied to naval structures subjected to dynamic shock loading could prove extremely beneficial for future shock survivability assessments performed using finite element modeling techniques.

\section{Experimental background}

In order to provide experimental data highlighting bolt behavior under a variety of load types, a series of experiments was carried out involving a bolt specimen subjected to different static and dynamic shear and tensile loading configurations [6]. These tests formed the basis for the numerical modeling that was the focus of the current research and data from these tests were used for validation of the numerical results presented later in this paper. The testing focused on bolts made of K-Monel K500, a corrosion-resistant nickel alloy commonly used in wetted naval applications, and with properties defined by QQ-N-286. Bolts in two lengths, $8.89 \mathrm{~cm}$ (3.5 in) for the shear tests, and $15.24 \mathrm{~cm}$ ( $6 \mathrm{in}$ ) for the tension tests, were manufactured according to MIL-S-1222G. The bolts of a given length were obtained from the same production lot to ensure consistency of the material properties between individual bolts. The bolts were drilled by Strainsert, using their standard process, to allow for instrumentation. Some of the bolts tested in the experiments presented in this paper were not actually instrumented, while others included strain gauges that were internally mounted on the inner surface of a blind bore hole. The instrumented bolts for the shear tests included a half bridge 350-ohm, type W gauge in the shear plane to measure shear strain, and the instrumented bolts for the tension tests included a full bridge, 120-ohm, type W strain gauge to measure tensile strain. All bolts had hex heads, were $6.35 \mathrm{~mm}(0.25 \mathrm{in})$ in diameter, and included a standard $1.9 \mathrm{~cm}(0.75 \mathrm{in})$ length of 20UNC-2A thread.

\subsection{Material testing}

The mechanical properties of the K500 bolt material were determined via quasistatic tensile testing of K500 bolts in accordance with ASTM E8 and ASTM F606. The mechanical and physical properties of the K500 bolt material are given in Table 1. Figure 1 shows the average stress-strain curve for the K500 material as determined from the tensile test data. Due to some discrepancies in the test data, it was necessary to piece together the strain data 


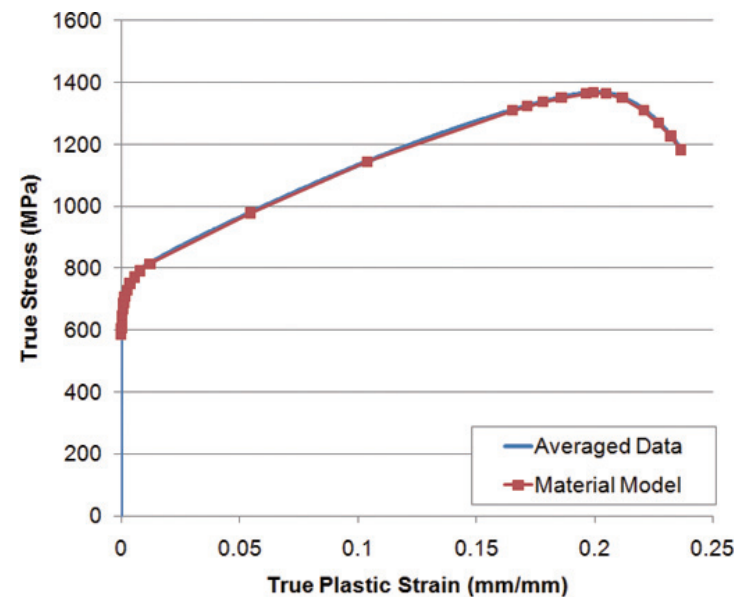

Fig. 1. Constitutive behavior of K-Monel K500 material.

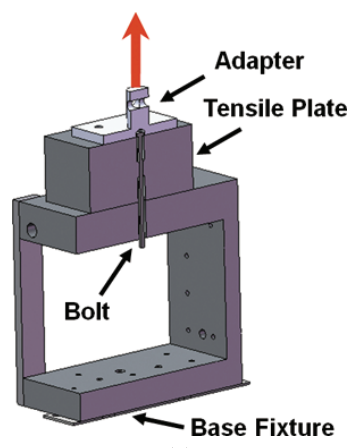

(a)

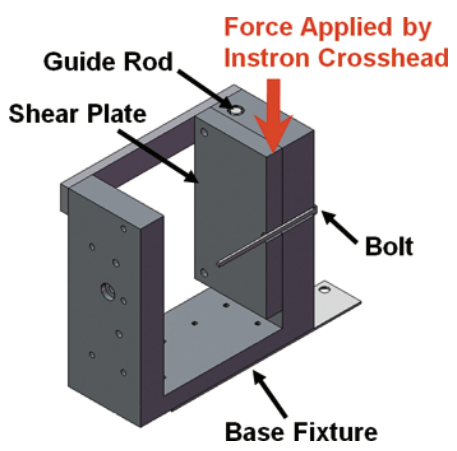

(b)

Fig. 2. Section view of (a) static tension test; (b) static shear test.

and displacement data to develop a single stress-strain curve for the material. This aggregate curve was developed by using the experimental strain data until just past the material yield (when the measured strain values started decreasing in a physically unrealistic manner), after which the experimental displacement data were used to calculate the strains corresponding to the remainder of the stress data points.

\subsection{Bolt testing}

In the main series of static and dynamic bolt experiments, a test bolt in a typical bolted connection was subjected to shear or tensile loading, as applied independently using a specially designed test fixture. Prior to loading, bolts were pre-tensioned to a force equivalent to $2 / 3$ of the yield stress for K500, consistent with standard practice for naval structures. Hardware for the experiment included an A36 steel base fixture, various A36 steel added mass and interface plates, high-strength steel guide rods, and the K500 shear and tensile bolt specimens. The bolts were tested to failure under quasistatic loading on an Instron 4206 machine, and under dynamic loading on a MIL-S-901 Lightweight Shock Machine (LWSM).

For the static tension tests, the tensile force was applied, by way of an adapter, to a tensile plate, as shown in Fig. 2(a). The bolt connected the tensile plate to the base fixture, which in turn was secured to the Instron machine. For the static shear tests, the test fixture was rotated 90 degrees from the static tension setup. The load was applied to the top center of the shear plate, which was free to move in the vertical direction along two lubricated guide rods, as per Fig. 2(b).

Two dynamic experimental test configurations were investigated on a Lightweight Shock Machine (LWSM), one focusing on tensile loading and the other on shear. For the dynamic tension tests, the bracket was mounted in the vertical plane of the LWSM interface plate so that the vertical hammer produced accelerations in the directions indicated in Fig. 3(a). For the dynamic shear tests, the test fixture was rotated 90 degrees from the dynamic tension setup, and the test fixture was mounted in the vertical plane on the LWSM interface plate so that the vertical hammer produced accelerations in the directions indicated in Fig. 3(b).

Although the four experimental configurations described above depict pure loading states, the authors realize that the loads actually imparted to the test bolts are not going to be restricted to pure tension or pure shear. However, each of these configurations was designed with an idealized situation in mind, and with the intent to capture a loading state as close as possible to a pure one. The approach taken towards simulating the actual loads applied to the bolts was to model the entire fixture and bolt in a highly detailed manner. In this way, although the bolt may not be subjected to pure tension or shear during the dynamic or static test, the loading actually applied to the bolt in the numerical model was as close as possible to the actual physical experiment. 


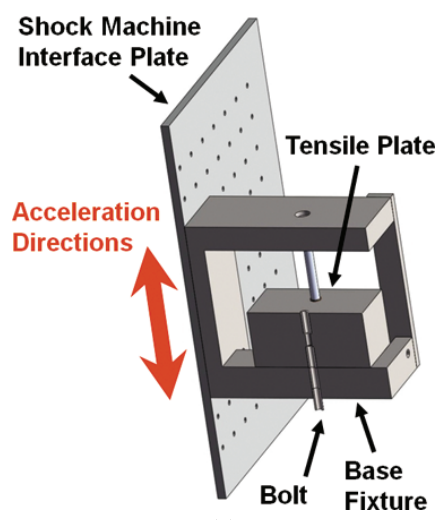

(a)

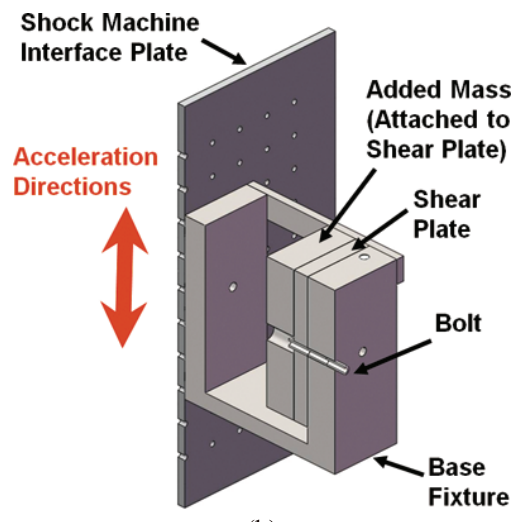

(b)

Fig. 3. Section view of (a) dynamic tension test; (b) dynamic shear test.

\section{Numerical modeling}

Finite element models of the previously described test hardware were developed using ABAQUS, version 6-9.1. All simulations were carried out in ABAQUS/Standard and ABAQUS/Explicit using double precision. C3D8 and C3D6 elements were used to model the shear and tensile bolts and their associated fixtures. Due to potential problems with accuracy, C3D6 elements were used sparingly, only in regions where irregular geometry warranted their use [18]. The numerical modeling focused on the subset of experiments in which the bolt specimens included a hollow bore, $3.9 \mathrm{~mm}(0.155 \mathrm{in})$ in diameter, for instrumentation, but which did not always include the instrumentation itself. All of the numerical simulations included a static preload to $2 / 3$ of the yield stress for K500, consistent with the experimental tests. All models included surface-to-surface contact between appropriate components. The surface-to-surface contact formulation imposes contact conditions in an average sense over regions near the slave nodes, where these regions are approximately centered on the slave nodes [1-3]. Finite sliding contact was used since the large amount of relative displacement between sliding components often seen in the experiments violates small sliding contact assumptions [1-3]. The models employed a standard penalty-based contact formulation, allowing for increased solver efficiency as compared with Lagrange multiplier-based alternative formulations [1-3]. The penalty method is a stiff approximation of hard contact, where the contact force is proportional to the penetration distance [1-3]. Per the software defaults, the ABAQUS/Standard models used a linear pressure-overclosure relationship with a penalty stiffness equal to 10 times the representative underlying element stiffness [1-3]. For the ABAQUS/Explicit models, the penalty stiffness that relates the contact force to the penetration distance was chosen automatically by the software to minimize the the effect on the stable time increment, per the software defaults [13]. Friction was included between all dry contact surfaces, with a static coefficient of friction of $\mu_{s}=0.78$, and a kinematic coefficient of friction of $\mu_{k}=0.42$. Additionally, all models used analysis methods that included the effects of nonlinear geometry, by using large-displacement formulations [1-5].

In the static models, the first stage of the analysis applied a pre-tightening force, equivalent to $2 / 3$ of the yield stress, to the bolt. This is achieved using the *PRE-TENSION SECTION, *SURFACE, and *STEP keywords, which together apply a prescribed force over the bolt cross-section in the direction of the bolt longitudinal axis [1, 2]. In the post-preload phase of the simulation, displacements were incrementally applied to the models and an arc length algorithm was used to obtain the corresponding nonlinear load-deflection response along its full path. Arc length methods are appropriate for simulations involving unstable, geometrically nonlinear collapse of a structure, or for investigating the post-buckling behavior of a structure [1-4]. The arc length method in ABAQUS/Standard follows the modified Riks algorithm [1-3,10,16], which is an efficient and widely used scheme to solve for the nonlinear equilibrium path for a structure under loading [4]. The modified Riks algorithm assumes that the loading is proportional, or that the applied load vector scales with a single scalar parameter, the load proportionality factor (LPF), which can take on positive or negative values. At each step, the increments in LPF and the displacement are unknown. The increment in displacement is obtained using the tangent stiffness matrix and the load for that step, while the increment in LPF is calculated using the current arc length, or path length in the solution space, and 


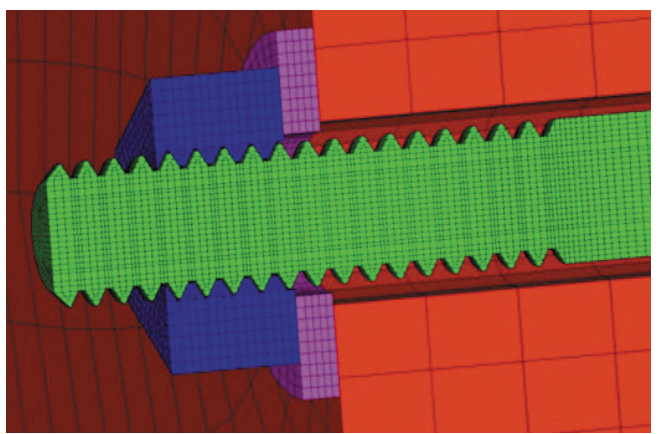

(a)

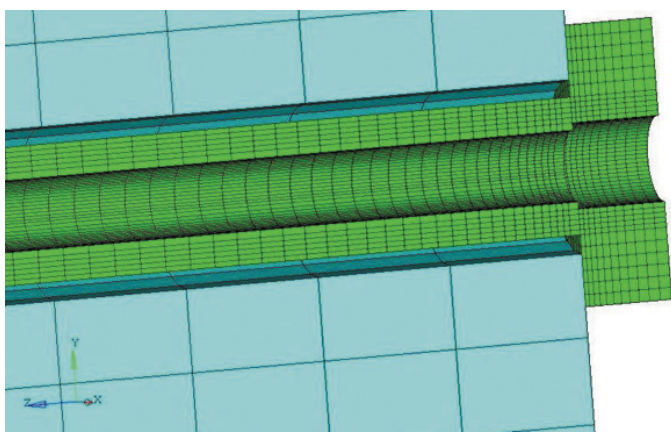

(b)

Fig. 4. Section views of static tension finite element model, details of (a) threaded end; and (b) bore and counterbore at head.

the current increment in displacement $[3,10,16]$. ABAQUS/Standard employs an automatic incrementation scheme where the arc length varies, and its values are bounded by user inputs for initial, minimum and maximum sizes [2, 3]. For the static analyses carried out for this research, parameter values used were an initial arc length of $1.27 \mathrm{~mm}$ (0.05 in), maximum length of $25.4 \mathrm{~mm}$ (1.0 in), and minimum length of $0.00254 \mathrm{~mm}$ ( $1 \mathrm{e}-4 \mathrm{in})$.

In the dynamic models, the bolt preload was accounted for through the application of an initial stress, equivalent to $2 / 3$ of the yield stress, to the bolt shank by way of the *INITIAL CONDITIONS keyword [1,2]. Explicit time integration was used to obtain the model response for the full duration of the simulated shock event. Automatic time stepping was used in order to maintain accuracy of the results, by way of keeping the time step sizes compatible with the conditional stability conditions that are associated with explicit methods [1-4].

\subsection{Static tests}

Physical observations of the static tension experiments indicated that the bolts all failed in the threaded region, and so care was taken to address the effect of the threads in these models. Based on the results from some preliminary studies, the threaded region was modeled using the correct profile for 20UNC-2A thread [15], but without the helical angle of the thread pattern - this modeling approach captured the effect of the reduced area in the threads without adding the complexity of producing a well-behaved mesh along a helically-varying thread profile. Additional preliminary analyses indicated that it was necessary to explicitly model the counterbore at the top of the hollow bore of the tensile bolt in order to improve accuracy of results. Figures 4(a) and (b) show details of the tension test bolt mesh, which included 484,500 degrees of freedom. The fixture, tensile plate, nut, and washer were modeled using a total of 274,200 degrees of freedom.

In the static tension models, contact between the fixture and tensile plate, fixture and bolt, tensile plate and bolt, washer and fixture, washer and nut, and bolt threads and nut was modeled using finite sliding contact. Displacements were incrementally applied to the top face of the tensile plate, and the load-deflection response was calculated using an arc length method. The static tension simulations were performed on four cores of a local HPC cluster, and required approximately 5.1 hours of wall clock time to fully trace the nonlinear equilibrium load-displacement path to a maximum displacement of $6.35 \mathrm{~mm}(0.25 \mathrm{in})$. Each core of the local HPC cluster was associated with a $2.67 \mathrm{GHz}$ Intel Xeon(R) processor and 32 GB RAM.

For the static shear tests, the test bolts were machined down $0.127 \mathrm{~mm}(0.005 \mathrm{in})$ after fabrication, producing a region $3.8 \mathrm{~mm}(0.15 \mathrm{in})$ long of reduced cross-sectional area in the vicinity of the shear plane, which was located at the interface between the sliding shear plate and the base fixture. As would be expected, in all of the experiments the test bolts failed in the reduced-area region of the bolt near this interface. Figure 5 includes the mesh detail of the test bolt at the shear plane for the static shear test arrangement. Since the bolts did not fail experimentally in the threads, the threads were not explicitly included in these models but rather the threaded region was represented using a reduced cross-sectional diameter equal to the average pitch diameter of the threads [15] over the threaded length of the bolt. The bolt was modeled with 319,500 degrees of freedom and the other components with 734,400 degrees of freedom. 


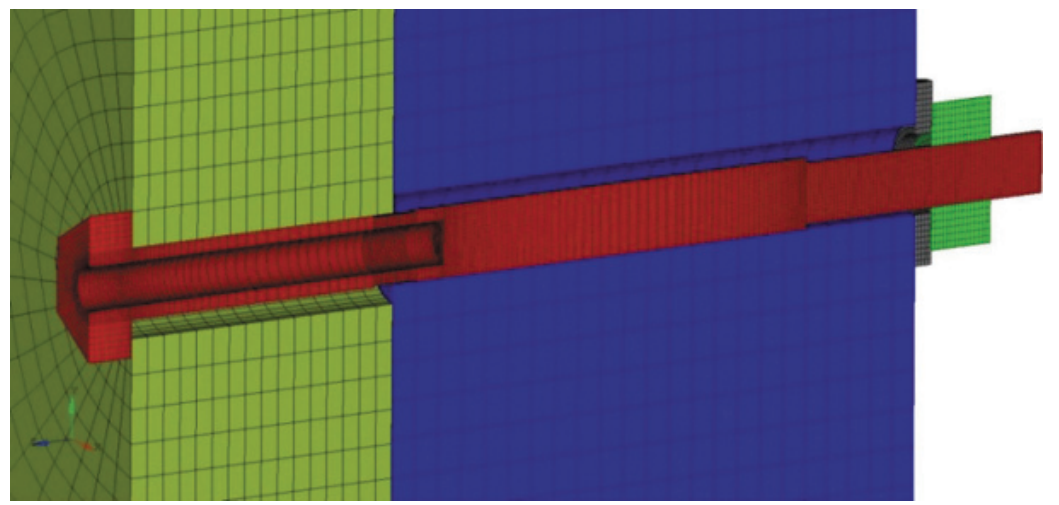

Fig. 5. Section view of finite element model, static shear test.

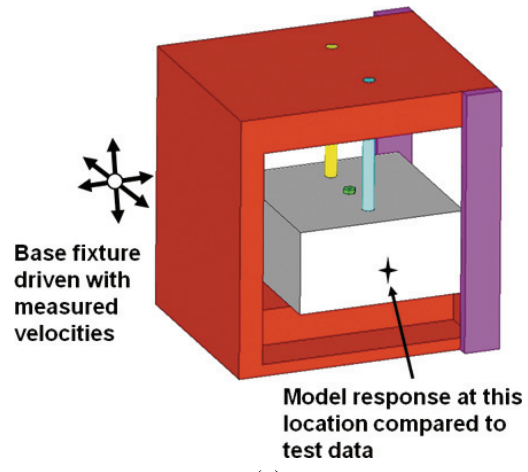

(a)

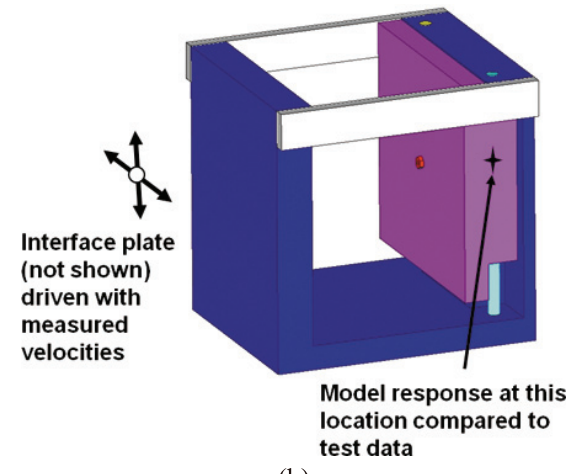

(b)

Fig. 6. Schematic of the model for (a) dynamic tension; and (b) dynamic shear.

Finite sliding contact was included between the guide rods and shear plate, fixture and shear plate, shear plate and bolt, fixture and bolt, washer and fixture, and washer and nut. The nut and bolt were constrained to move together. Displacements were incrementally applied to the top and bottom faces of the shear plate, and the nonlinear load-deflection response was obtained using an arc length algorithm. As with the static tension analyses, the static shear simulations were carried out on four cores of a local HPC cluster. However, due to the amount of contact and complexity of failure, the static shear analyses required more run time than the static tension simulations, using approximately 31.3 hours of wall clock time to fully trace the nonlinear equilibrium path to a maximum displacement of $2.29 \mathrm{~mm}(0.09 \mathrm{in})$.

\subsection{Dynamic tests}

The simulations of the dynamic tension and shear tests were performed on the SGI Altix ICE (Diamond) HPC cluster at the US Army Engineer Research and Development Center (ERDC). The dynamic models were driven with velocity time histories, where these time histories had been obtained from triaxial accelerometer data recorded on the LWSM interface plate. This interface plate was connected to the base fixture during the dynamic tension and shear experiments. Schematics of the models are shown in Figs 6(a) and (b). Note that for the dynamic shear model, the out-of-plane response of the shear test fixture was accounted for by modeling the interface plate explicitly. Thus the dynamic shear model was driven with only the in-plane and vertical components of velocity at the edges of the interface plate.

Since the specimens of the K500 material were only tested under quasistatic conditions in order to characterize the behavior of the material, the rate-dependent behavior of the bolt material actually used in the physical experimental tests was unknown. Two analyses were performed for each dynamic load configuration, one with no rate-dependence 


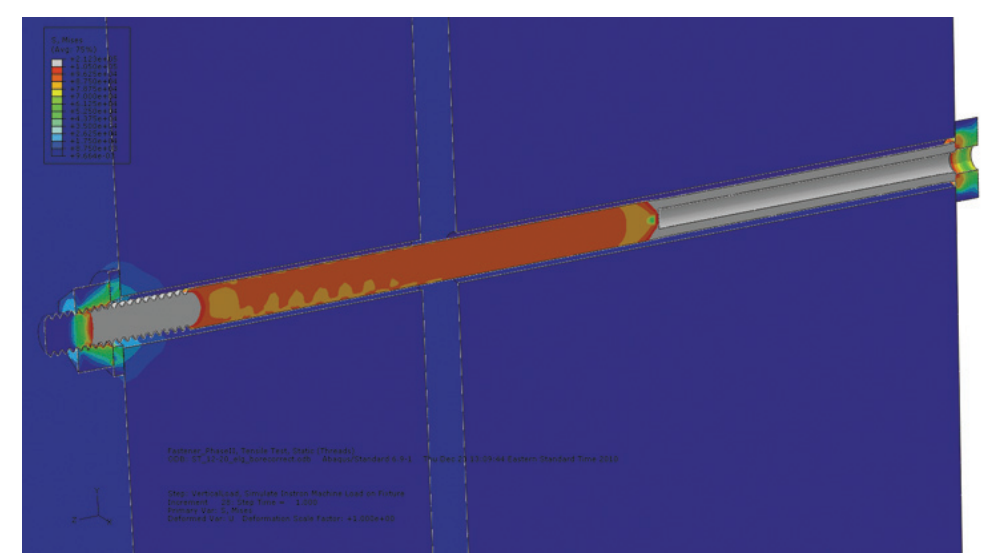

Fig. 7. Contours of von Mises stresses at maximum applied displacement, static tension model.

in the material model and another with an estimate of rate-dependent material properties. For the latter analyses, a dynamic increase factor of $30 \%$ was globally applied to the stress values of the K500 quasistatic stress-strain curve in order to approximate the K500 constitutive behavior at a strain rate of 1000/s. This level of rate-dependence was assumed as an upper bound in possible rate-dependent material effects for the K-Monel K500. Thus, results from these two analyses were intended to bracket the range of behavior originating from the (unknown) rate-dependent material effects.

For the dynamic tension models, the threaded region was again modeled using the correct profile for a 20UNC-2A thread [15] without the helically-varying thread profile. Finite sliding contact was included between the guide rods and tensile sliding mass, fixture and sliding mass, fixture and bolt, sliding mass and bolt, washer and fixture, washer and nut, and bolt threads and nut. Contact between components was enforced using a penalty-based algorithm, as suggested by other research [18]. The bolt was modeled with 439,000 degrees of freedom and the fixture and other associated test components with 581,400 degrees of freedom. The dynamic tension simulations were performed on 32 cores of the ERDC Diamond cluster, and required about 66 hours of wall clock time to produce 76 ms of response, which corresponds to a rate of 0.9 hours per ms of data.

For the dynamic shear models, the threaded region was again represented using a circular cross-section with a reduced diameter equal to the average pitch diameter [15] of the 20UNC-2A thread. Finite sliding contact, enforced by way of a penalty-based algorithm, was included between the guide rods and shear plate, fixture and shear plate, shear plate and bolt, fixture and bolt, washer and fixture, and washer and nut. The nut and bolt were constrained to move together. The added sliding mass, which was attached to the main sliding shear plate in the experiments, was modeled by way of lumped masses attached to the shear plate at each of the four bolted locations. Preliminary analyses showed that, in order to achieve accurate results for the dynamic shear modeling, it was necessary to explicitly model the LWSM interface plate and apply the dynamic loads to the interface plate, rather than use the time histories measured at the interface plate to drive the fixture [7]. Essentially, the LWSM interface plate did not act like a rigid body and so its response to the dynamic loading was necessary in order to correctly capture the loading on the fixture. Accordingly, the experimentally recorded accelerometer data was integrated and applied as velocity time histories in the vertical and in-plane directions at the corresponding accelerometer location on the LWSM interface plate model.

Preliminary analyses indicated that it was necessary to include some type of failure criterion in the static and dynamic shear models because the experimentally observed shearing of the bolts had a large effect on the overall structural response to the static and dynamic loading. Accordingly, in the dynamic analyses, the fracture associated with the shear failure of the bolt was modeled using a strain-to-failure criterion (*SHEAR FAILURE), whereby elements were deleted from the model when shear strains reached a given level [2], prescribed herein as $0.24 \mathrm{~mm} / \mathrm{mm}$. Element deletion based on strain-to-failure is only available within ABAQUS/Explicit, and so an alternative approach was used for the static shear analysis, carried out in ABAQUS/Standard. This approach models damage by progressive degradation of the material stiffness, which ultimately leads to material failure [1-3]. The approach is included in a model by using the *DAMAGE_INITIATION and *DAMAGE_EVOLUTION keywords [2]. Damage 


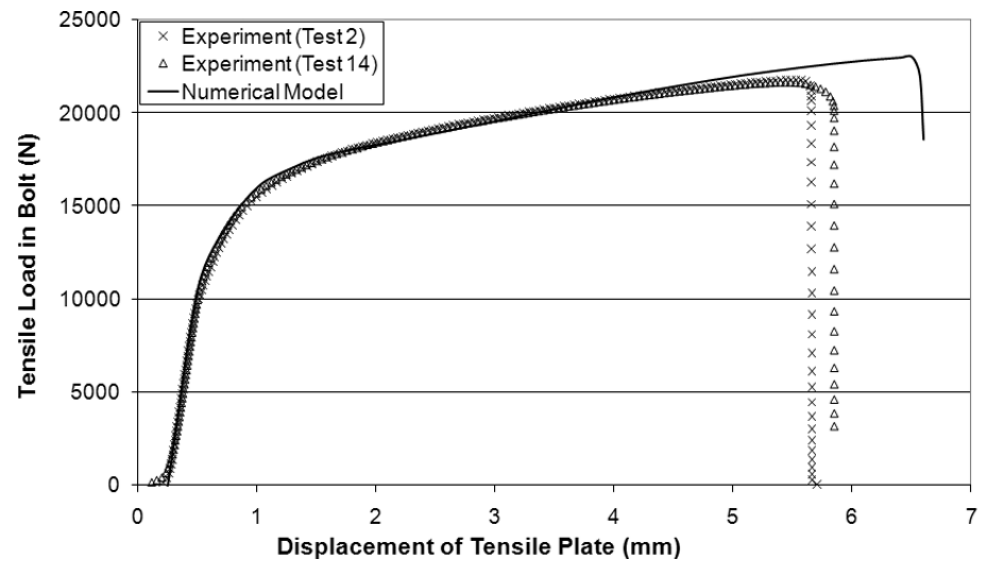

Fig. 8. Comparison with experimental load-displacement data, static tension test.

is initiated at a plastic strain of $0.20 \mathrm{~mm} / \mathrm{mm}$, corresponding to the peak in the stress-plastic strain curve, shown in Fig. 1, and the evolution of damage is a function of the plastic displacement measured after the initiation of damage, $u^{p l}$ [1-3]. From the material test data [6], the amount of plastic displacement corresponding to failure is $1.14 \mathrm{~mm}$ ( 0.045 in) for the K-Monel K500 used in the static shear experiments. The bolt model included 292,600 degrees of freedom and the fixture and other components consisted of 917,000 degrees of freedom. The dynamic shear simulations were performed on 32 cores of the ERDC Diamond cluster, and required about 69 hours of wall clock time to produce $56 \mathrm{~ms}$ of response. This corresponds to a rate of 1.2 hours per ms of data, which is more computationally expensive than the dynamic tension models, again because of the complexity of the contact and also the material failure involved in this problem.

\section{Results and discussion}

Both qualitative and quantitative comparisons were made between numerical models and the physical experiments for the different load configurations tested. For the static tests, the qualitative comparisons consisted of observations regarding the location and progression of failure in the bolt specimens, and the quantitative comparisons were made between load-displacement data as measured experimentally and numerically. Additionally, for the static tests, limited strain data were available from a few of the instrumented bolts; as practicable, quantitative comparisons are made between the experimental strain data and the appropriate strain components measured at the corresponding gauge locations in the numerical models.

\subsection{Static tests}

In the static tension tests, the bolts experienced high levels of deformation in the reduced-area region of the hollow bore (slight necking) and in the threads, where the bolts ultimately failed. These same regions of high stress are also seen in the results of the finite element simulations, as shown in Fig. 7. The von Mises stress contours here are given in units of pounds per square inch, spanning from 0 psi to 212,300 psi (1464 MPa), in increments of 8,750 psi (60.3 MPa).

The load-displacement data from the static tension experiments compare extremely well to the corresponding load-displacement data from the finite element simulations, as shown in Fig. 8. The experimental load levels and displacements were measured at the Instron machine crosshead, and were compared to the vertical nodal displacements applied to the nodes on the top face of the tensile plate and the corresponding forces developed there. The tensile plate was attached to the Instron crosshead by way of an adapter, as seen in Fig. 2(a).

A schematic of the strain gauge placement for the static tension experiments is shown in Fig. 9. Note that although the bolts failed experimentally at the threads, it is not practical to instrument this region of a bolt, and so strains 


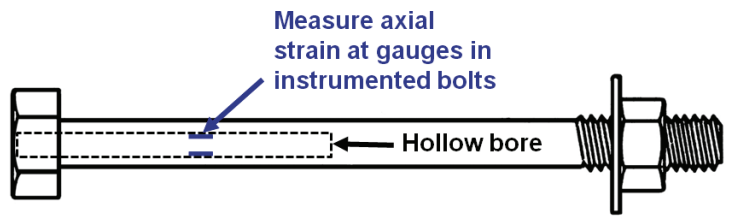

Fig. 9. Schematic of strain gauge placement, static tension test.

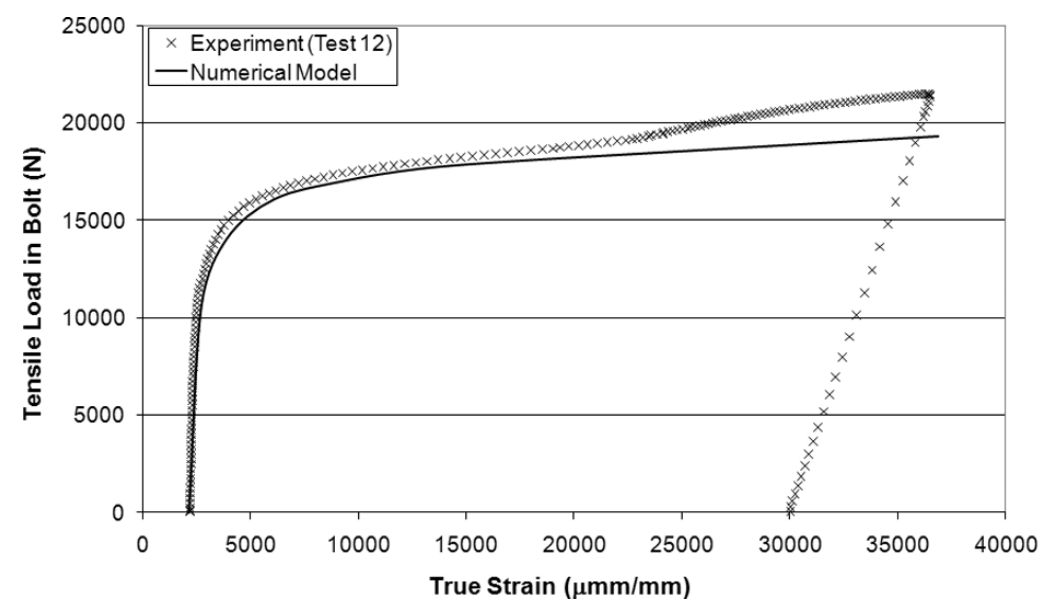

Fig. 10. Comparison with experimental load-strain data, static tension test.

were measured in the bolt body away from the ultimate failure location. Strain results in the axial direction are extracted from the gauge locations in the numerical model and are compared to the tensile strain data from an experiment in Fig. 10. As was common with all of the instrumented experiments, the strain gauges ultimately debonded prematurely. It can be seen that for the experimental data presented here, the debonding initiated at a strain level of approximately 22,000 microstrains, as evidenced by the increased slope of the load-strain data at this point, and then the gauge completely debonded at 35,000 microstrains. Despite the ultimate failure of the strain gauge, there is excellent agreement between the numerical and experimental results up to the point of initial debonding.

For the static shear experiments, the bolts experienced the highest levels of deformation near the shear interface, where the bolts ultimately failed. This is also observed in the numerical results, as seen in Fig. 11. In Fig. 11(a), the von Mises stress contours are shown in units of pounds per square inch, spanning from 0 psi to 147,100 psi $(1,014 \mathrm{MPa})$, in increments of 16,350 psi (112.7 MPa). Figure 11(b) shows the equivalent plastic strain, in increments of $0.029 \mathrm{~mm} / \mathrm{mm}$, spanning from 0 to $0.257 \mathrm{~mm} / \mathrm{mm}$. Note that per the damage model used in this analysis, damage via progressive degradation of material stiffness initiates at an equivalent plastic strain value of $0.20 \mathrm{~mm} / \mathrm{mm}$.

A comparison of the load-displacement data from the static shear experiments and the finite element simulation results is depicted in Fig. 12. The experimental load levels and displacements at the Instron machine crosshead were compared to the vertical nodal displacements applied to the finite element nodes located at the top and bottom faces of the shear plate and corresponding forces developed at those locations. The Instron crosshead was in direct contact with the top of the shear plate, and a schematic of the setup is pictured in Fig. 2(b). Due to the more complex nature of the shear loading, there is more scatter in the experimental data as compared to the static tension results. However, the numerical results match the general trend of the experimental data fairly well. Differences between the numerical and experimental results can be attributed to the approximation of shear failure provided by the numerical damage model as compared with the physical failure mechanisms acting within the actual bolt.

The typical strain gauge placement for the static shear experiments is depicted in Fig. 13. Unlike the static tension experiments, the gauges are placed at the actual location of failure for the static shear experiments, giving a very local measurement of failure. Figure 14 includes a comparison of the shear strain data from the experiments and the numerical model. Again, the strain gauges ultimately debonded prematurely. For the available experimental data, debonding occurred at strain levels around 3,000 microstrains, although for one test the gauge lasted up to 


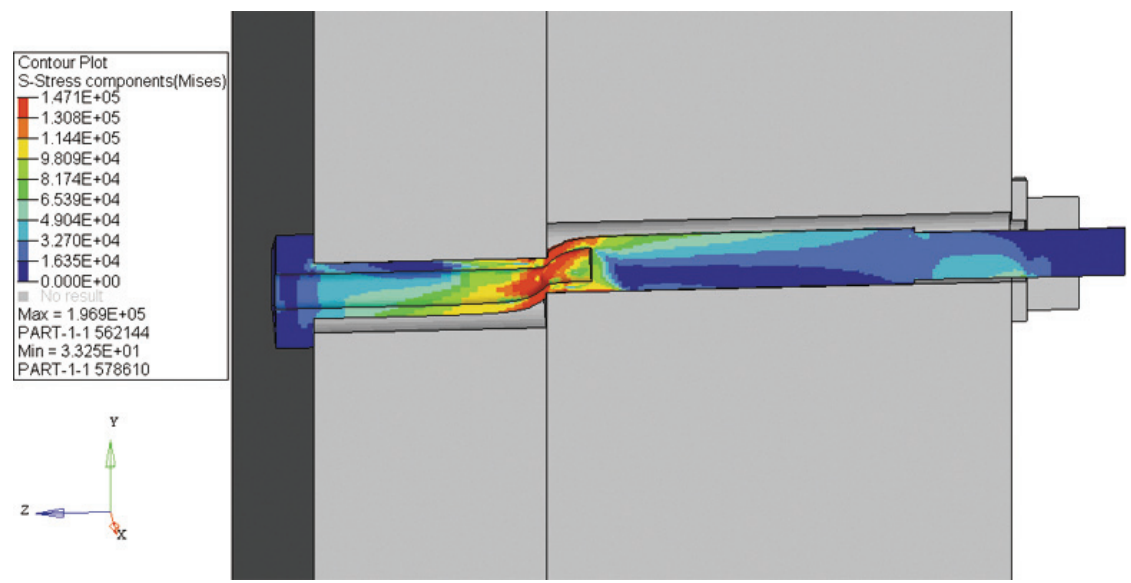

(a)

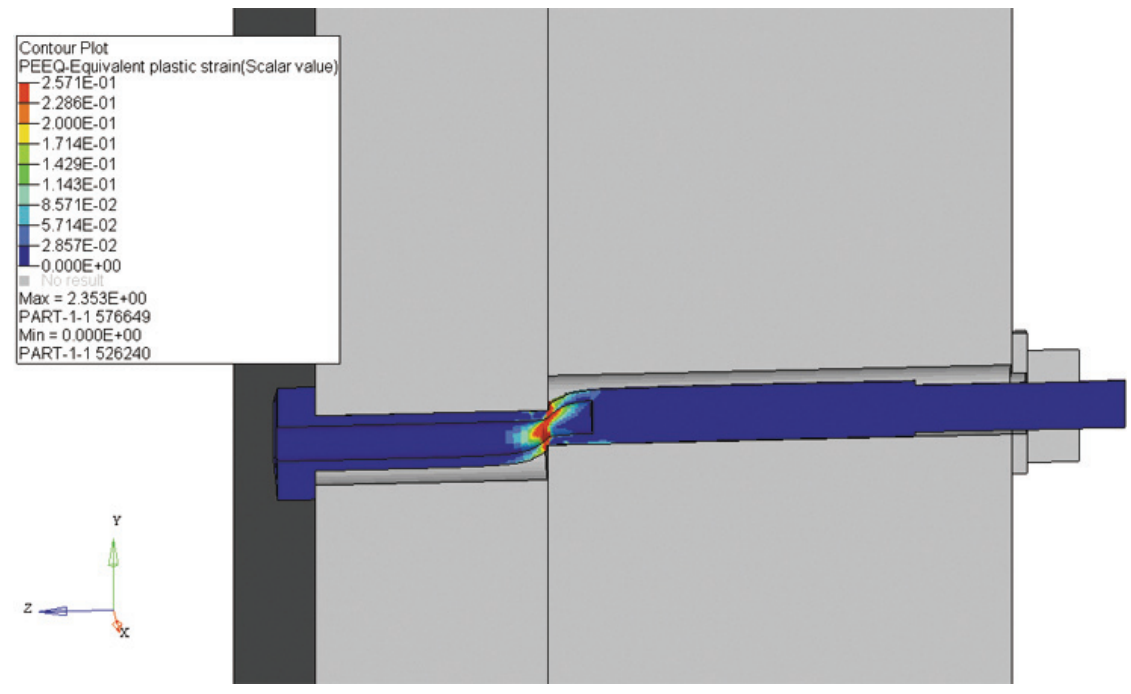

(b)

Fig. 11. Contours of (a) von Mises stresses and (b) equivalent plastic strain, at maximum applied displacement, static shear model.

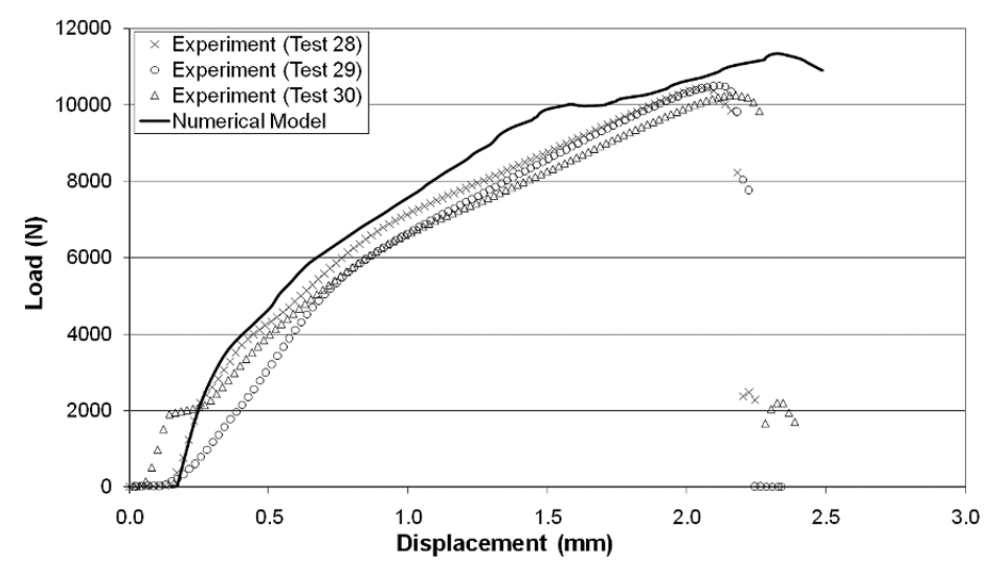

Fig. 12. Comparison with experimental load-displacement data, static shear test. 


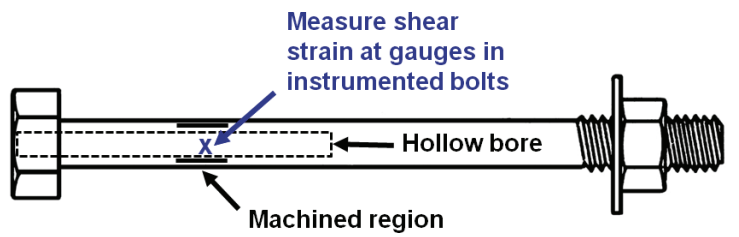

Fig. 13. Schematic of strain gauge placement, static shear test.

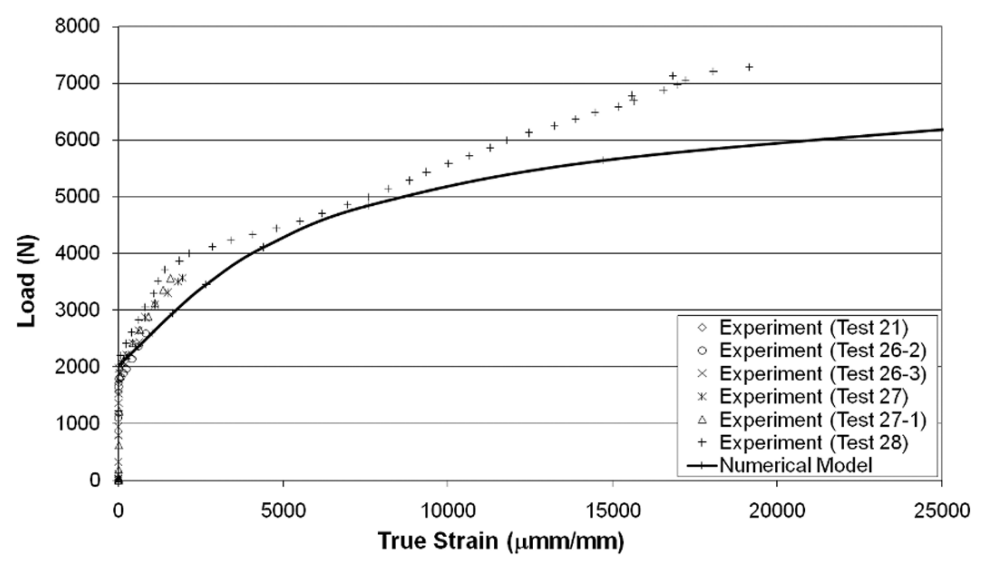

Fig. 14. Comparison with experimental load-strain data, static tension test.

20,000 microstrains. Although there appears to be generally good agreement between the numerical and experimental results for this data set, the authors are unsure of the quality of the data past 3,000 microstrains, given the debonding problems inherent in all of the instrumented static shear experiments.

\subsection{Dynamic tests}

In the dynamic tension experiments, the bolts experienced the highest levels of deformation in the threads, where the bolts ultimately failed. This same trend in deformation is also seen in the finite element simulation results. Figure 15 depicts the contours of equivalent plastic strain at a time $24 \mathrm{~ms}$ into the analysis, where the contours span from 0 to $0.13 \mathrm{~mm} / \mathrm{mm}$ in increments of $0.01 \mathrm{~mm} / \mathrm{mm}$.

The velocity data that were experimentally recorded at the tensile sliding mass, at the location indicated in Fig. 6(a), compare well with the corresponding data from the finite element simulations. The experimental and numerical results are plotted together in Fig. 16. Generally, it was found that the rate-dependence of the material model did not have a significant effect on the general velocity results, as shown by the similarity of the velocity time histories in the rate-independent and rate-dependent analyses, shown in Fig. 17.

In the dynamic shear tests, the bolts experienced the highest amounts of plastic deformation near the interface between the shear sliding mass and the base fixture, eventually shearing apart there. The same concentration of bolt deformation near the shear plane is seen in the numerical results, as shown in Fig. 18 at a time 22 ms into the analysis. At this point in the analysis, the bolt is just starting to shear, according to the specified failure criterion. The contours of equivalent plastic strain range from 0 to $0.24 \mathrm{~mm} / \mathrm{mm}$, and are shown in increments of $0.02 \mathrm{~mm} / \mathrm{mm}$.

The velocity data experimentally recorded at the tensile sliding mass, as per Fig. 6(b), is plotted together with the finite element simulation results in Fig. 19. The velocity data from the numerical models compares well with the experimental data at the corresponding gauge location.

For the dynamic shear results, the rate-dependence of the material model had almost no effect at all on the velocity results at the accelerometer location, as shown in Fig. 20. This is physically logical, given that the structural response in both models is dominated by a complete shearing of the bolt at a time of $22 \mathrm{~ms}$ into the analysis, an effect which is captured by the material failure criterion. 


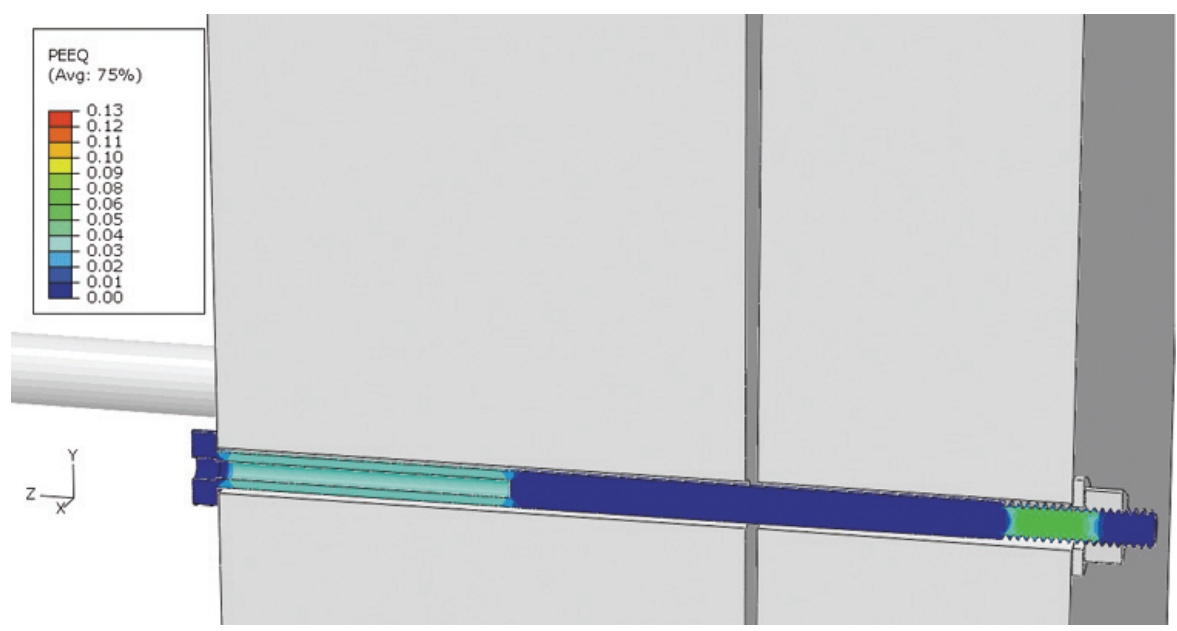

Fig. 15. Contours of equivalent plastic strain, dynamic tension model, $t=24 \mathrm{~ms}$.

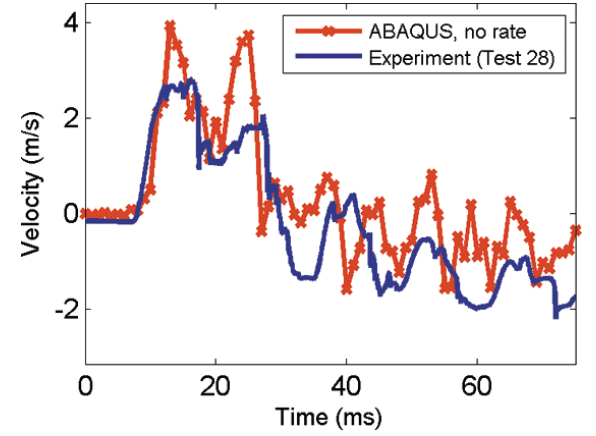

Fig. 16. Comparison with experimental velocity data, dynamic tension test.

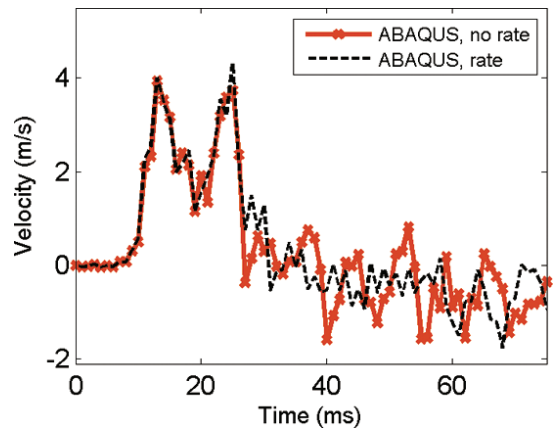

Fig. 17. Comparison between rate-independent and rate-dependent model results, dynamic tension test.

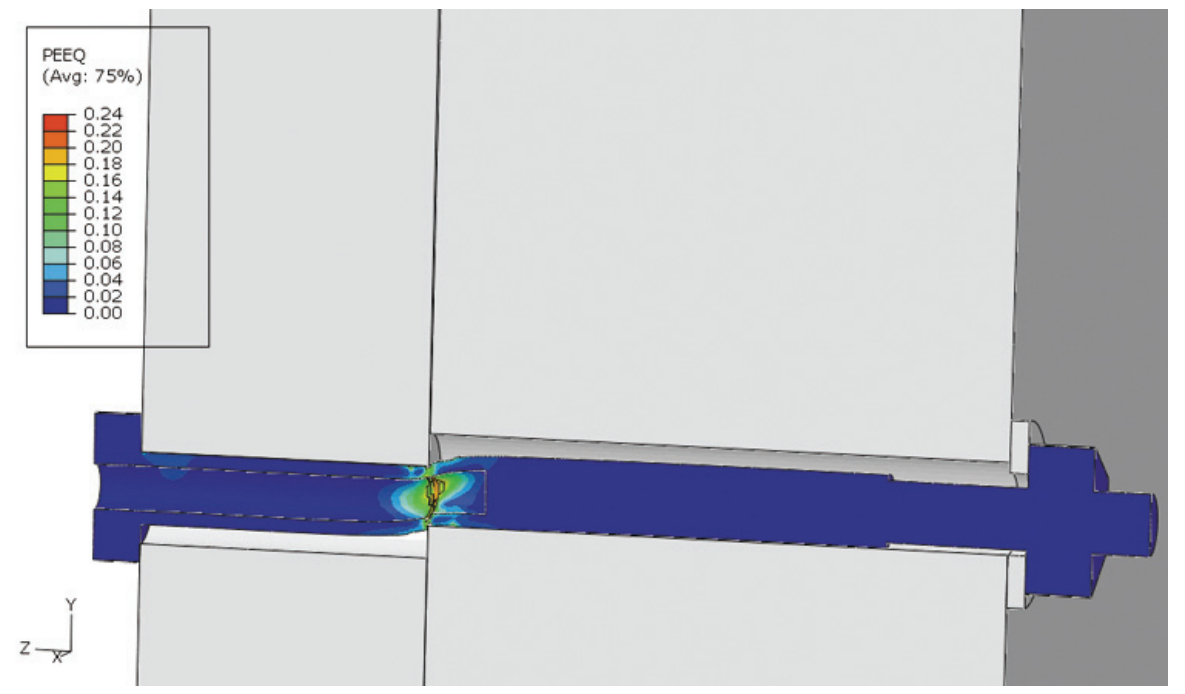

Fig. 18. Contours of equivalent plastic strain, dynamic shear model, $t=22 \mathrm{~ms}$. 
Table 2

Russell comprehensive error $(\mathrm{RC})$ results

\begin{tabular}{lclc}
\hline Case & Measured data, $m_{i}$ & Calculated data, $c_{i}$ & $\mathrm{RC}$ \\
\hline Dynamic tension & Experiment & ABAQUS, no rate & 0.204 \\
Dynamic tension & Experiment & ABAQUS, rate & 0.197 \\
Dynamic shear & Experiment & ABAQUS, no rate & 0.116 \\
Dynamic shear & Experiment & ABAQUS, rate & 0.115 \\
\hline
\end{tabular}

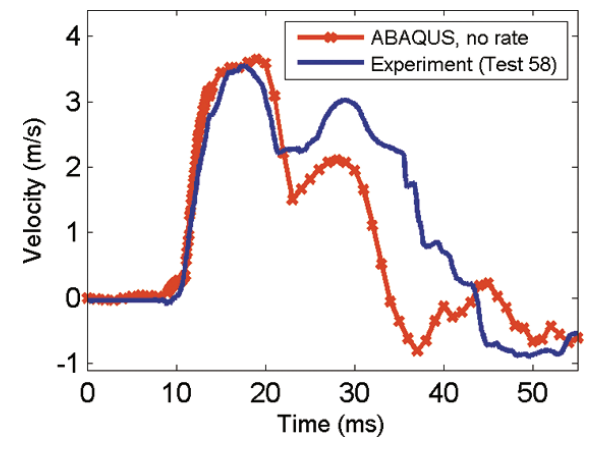

Fig. 19. Comparison with experimental velocity data, dynamic shear test.

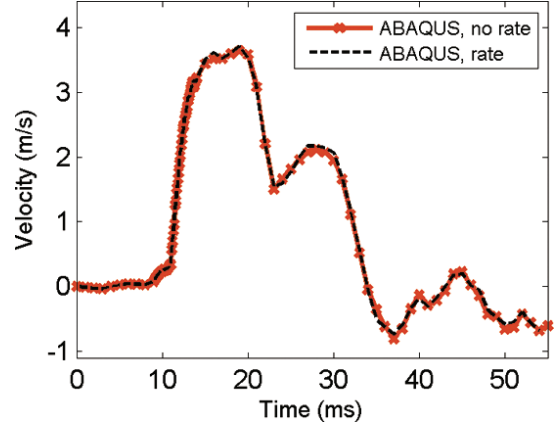

Fig. 20. Comparison between rate-independent and rate-dependent model results, dynamic shear test.

\subsection{Russell comprehensive error}

The correlation between the experimental and numerical data can be quantified via the Russell comprehensive error metric. Russell error is one method for comparing two transient data sets, where variations in magnitude and phase are both quantified, and then these differences are combined into a single error metric, known as the comprehensive error. The phase (RP), magnitude (RM), and comprehensive (RC) Russell error metrics are calculated as follows [17]:

$$
\begin{aligned}
R P & =\frac{1}{\pi} \cos ^{-1}\left(\frac{\sum c_{i} m_{i}}{\sqrt{\sum c_{i}^{2} \sum m_{i}^{2}}}\right) \\
R M & =\operatorname{sign}(m) \log _{10}(1+|m|) \\
R C & =\sqrt{\frac{\pi}{4}\left(R M^{2}+R P^{2}\right)}
\end{aligned}
$$

In Eqs (1)-(3), $c_{i}$ and $m_{i}$ represent the calculated and measured responses, respectively. Correlation between transient data sets is subjectively classified as 'excellent', 'acceptable', or 'poor', where these levels are defined as: Excellent $-\mathrm{RC}<=0.15$, Acceptable $-0.15<\mathrm{RC}<=0.28$, and Poor $-\mathrm{RC}>0.28$. The delineation between criterion levels resulted from a study that was carried out to calibrate opinions within a team examining velocity data from a ship shock trial. In the context of the research presented in this paper, the calculated responses correspond to numerical data from finite element analyses, and the measured responses correspond to experimental data.

The Russell comprehensive error metric was calculated for both the dynamic tension and dynamic shear tests. Comparisons are made between the experimental data and the numerical data from both rate-independent and ratedependent analyses. Results are summarized in Table 2, and show that the numerical models produce excellent correlation for the dynamic shear tests and acceptable correlation for the dynamic tension tests.

\section{Conclusions}

In this paper, an approach is presented for high-resolution finite element modeling of K-Monel K500 bolts in static and dynamic tension and shear configurations. The four load configurations represent idealized load cases; 
the approach taken towards simulating the actual loads applied to the bolts was to model the entire fixture and bolt in a highly detailed manner so that the loading actually applied to the bolt in the numerical model was as close as possible to the actual physical experiment, regardless of purity of load state. Modeling approaches for each load type varied, with special attention taken to include key details from the experiments that were necessary in order to adequately capture the appropriate physics. This involved the inclusion of a failure criterion for the K500 material in the shear tests, as well as explicit modeling of (1) the bolt threads for the tensile loading arrangements, (2) the counterbore at the top of the hollow bore of the tensile bolt in the static tension tests, and (3) the Lightweight Shock Machine (LWSM) interface plate in the dynamic shear test. The best results for the static cases were achieved using displacement-based control, rather than load-based control, and finite sliding contact without automatic stabilization.

Due to a lack of dynamic material data for K500, the authors chose to bound the (unknown) rate-dependent behavior of the K500 material by carrying out two analyses for each dynamic configuration, one with rate-independent material behavior and the other with rate-dependent constitutive relationships. For the latter, the constitutive behavior at a high strain rate $(1,000 / \mathrm{s})$ was estimated by applying a dynamic increase factor of $30 \%$ to the stress values of the K500 quasistatic stress-strain curve. The results of these analyses showed that, for the loading configurations examined in this research, the rate-dependent material behavior appears to have a small effect in the overall structural response.

Numerical results for all four load configurations - static tension, static shear, dynamic tension, and dynamic shear - compare well with experimental data. For the static tests, both global and local measures were available for comparison of structural response, in the form of load-displacement data (global) and limited strain data, recorded from internally mounted strain gauges in the bolt (local). However, capturing strain data proved extremely difficult due to debonding issues with the strain gauges and the severity of the loading conditions, so the quality of the strain data was not very good and no strain data were available for the dynamic tests. However, quantitative comparisons were made to velocity data for the dynamic tests. Using the Russell comprehensive error metric, there is acceptable correlation between the experimental and the numerical velocity data for the dynamic tension model, and excellent correlation between the velocity data at the experimental accelerometer location for the dynamic shear model. Future research can build on these validated models, and focus on developing simplified finite element bolt representations and calibrating them to the experimental data for the four load configurations.

\section{Acknowledgments}

This work was supported by internal basic and applied research funding (NUWCDIVNPT). The DoD HPC Modernization Program supported this project by supplying supercomputer time at the US Army Engineer Research and Development Center. We would like to acknowledge Pierre Corriveau, Tony Ruffa, Dawn Vaillancourt, and the entire Chief Technology Office at NUWCDIVNPT.

\section{References}

[1] Abaqus 6.9 Analysis User's Manual, vol. 1-5, Dassault Systems, USA, 2009.

[2] Abaqus 6.9 Keywords Manual, vol. 1-2, Dassault Systems, USA, 2009.

[3] Abaqus 6.9 Theory Manual, Dassault Systems, USA, 2009.

[4] K.-J. Bathe, Finite Element Procedures, Prentice Hall, New Jersey, 1996.

[5] T. Belytschko, W.K. Liu and B. Moran, Nonlinear Finite Elements for Continua and Structures, John Wiley \& Sons, Ltd., New York, 2000 .

[6] K.E. Behan, C.P. Pabon, E.L. Guzas, J.R. Milburn and S.M. Moss, Results of the static and dynamic testing for the improved fastener modeling: Phase II testing, Technical Report No. NUWC-NPT-TM2010/100, 2010.

[7] K.E. Behan, E.L. Guzas, J.R. Milburn and S.M. Moss, Finite element modeling of fasteners under static and dynamic loading, Proceedings of the High Performance Computing Modernization Program (HPCMP) Users Group Conference, Portland, OR, (20-23 June 2011).

[8] K.F. Chung and K.H. Ip, Finite element modeling of bolted connections between cold-formed steel strips and hot rolled steel plates under static shear loading, Engineering Structures 22(10) (2000), 1271-1284.

[9] K.F. Chung and K.H. Ip, Finite element investigation on the structural behavior of cold-formed steel bolted connections, Engineering Structures 23(9) (2001), 1115-1125.

[10] M.A. Crisfield, A fast incremental/iterative solution procedure that handles snap-through, Computers \& Structures 13(1-3) (1981), 55-62. 
[11] K. Khandelwal, S. El-Tawil, S.K. Kunnath and H.S. Lew, Macromodel-based simulation of progressive collapse: Steel frame structures, Journal of Structural Engineering 134(7) (2008), 1070-1078.

[12] K. Khandelwal, S. El-Tawil and F. Sadek, Progressive collapse of seismically designed steel braced frames, Journal of Constructional Steel Research 65(3) (2009), 699-708.

[13] M.A. McCarthy, C.T. McCarthy, V.P. Lawlor and W.F. Stanley, Three-dimensional finite element analysis of single-bolt, single-lap composite bolted joints: Part I - model development and validation, Composite Structures 71(2) (2005), 140-158.

[14] C.T. McCarthy and M.A. McCarthy, Three-dimensional finite element analysis of single-bolt, single-lap composite bolted joints: Part II effects of bolt-hole clearance, Composite Structures 71(2) (2005), 159-175.

[15] E. Oberg, F.D. Jones and H.L. Horton, Machinery's Handbook, 23rd edition, Industrial Press Inc., New York, 1988.

[16] E. Ramm, Strategies for tracing nonlinear responses near limit points, Nonlinear Finite Element Analysis in Structural Mechanics, W. Wunderlich, E. Stein and K.J. Bathe, eds, Springer-Verlag, New York, 1981, pp. 63-89.

[17] D.M. Russell, Error measures for comparing transient data, part I: Development of a comprehensive error measure, part II: Error measures case study, Proceedings of the 68th Shock and Vibration Symposium, Hunt Valley, MD, (3-6 November 1997).

[18] S. Selamet and M.E. Garlock, Guidelines for modeling three dimensional structural connection models using finite element methods, Proceedings of the International Symposium "Steel Structures: Culture and Sustainability”, Istanbul, Turkey, (21-23 September 2010). 

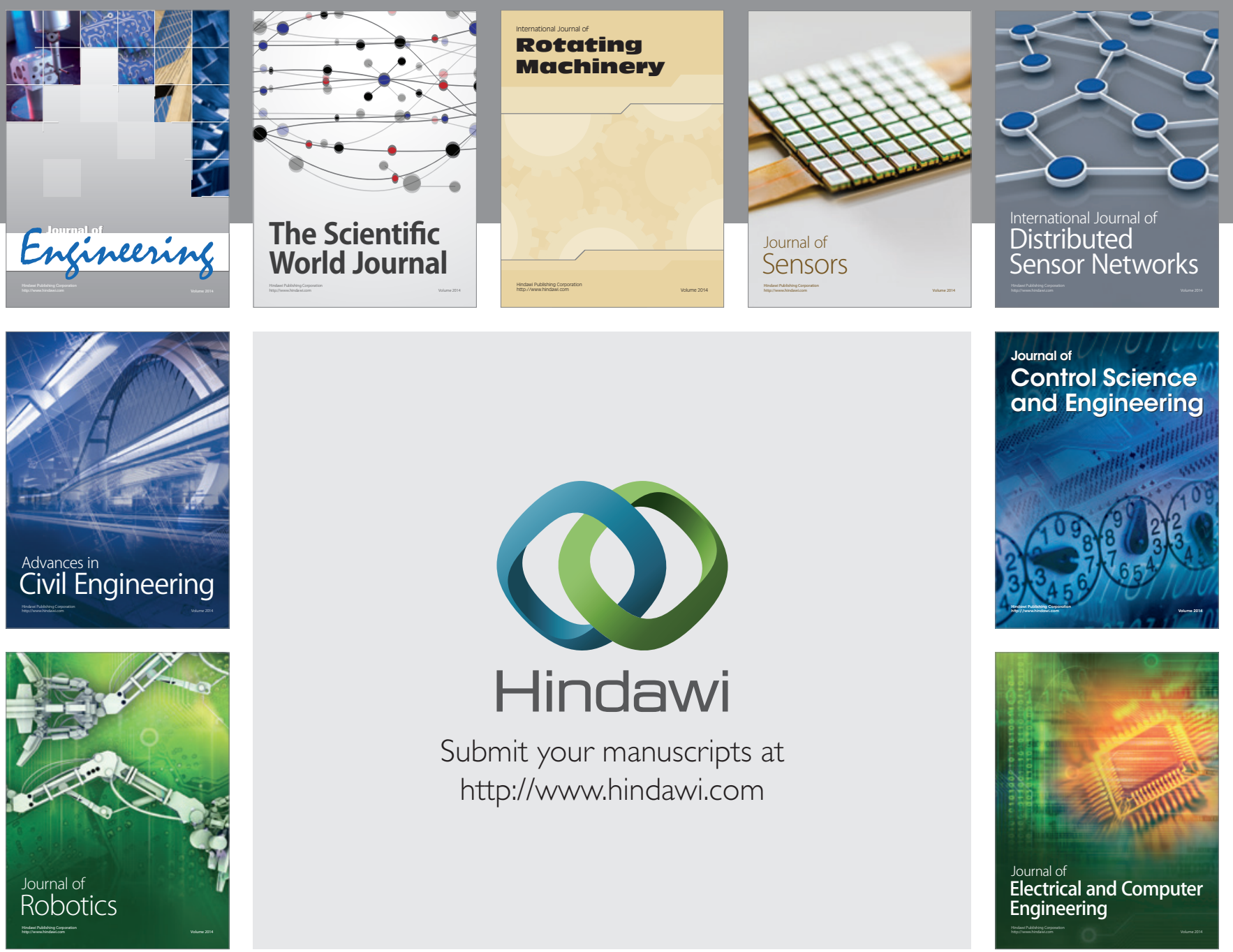

Submit your manuscripts at

http://www.hindawi.com
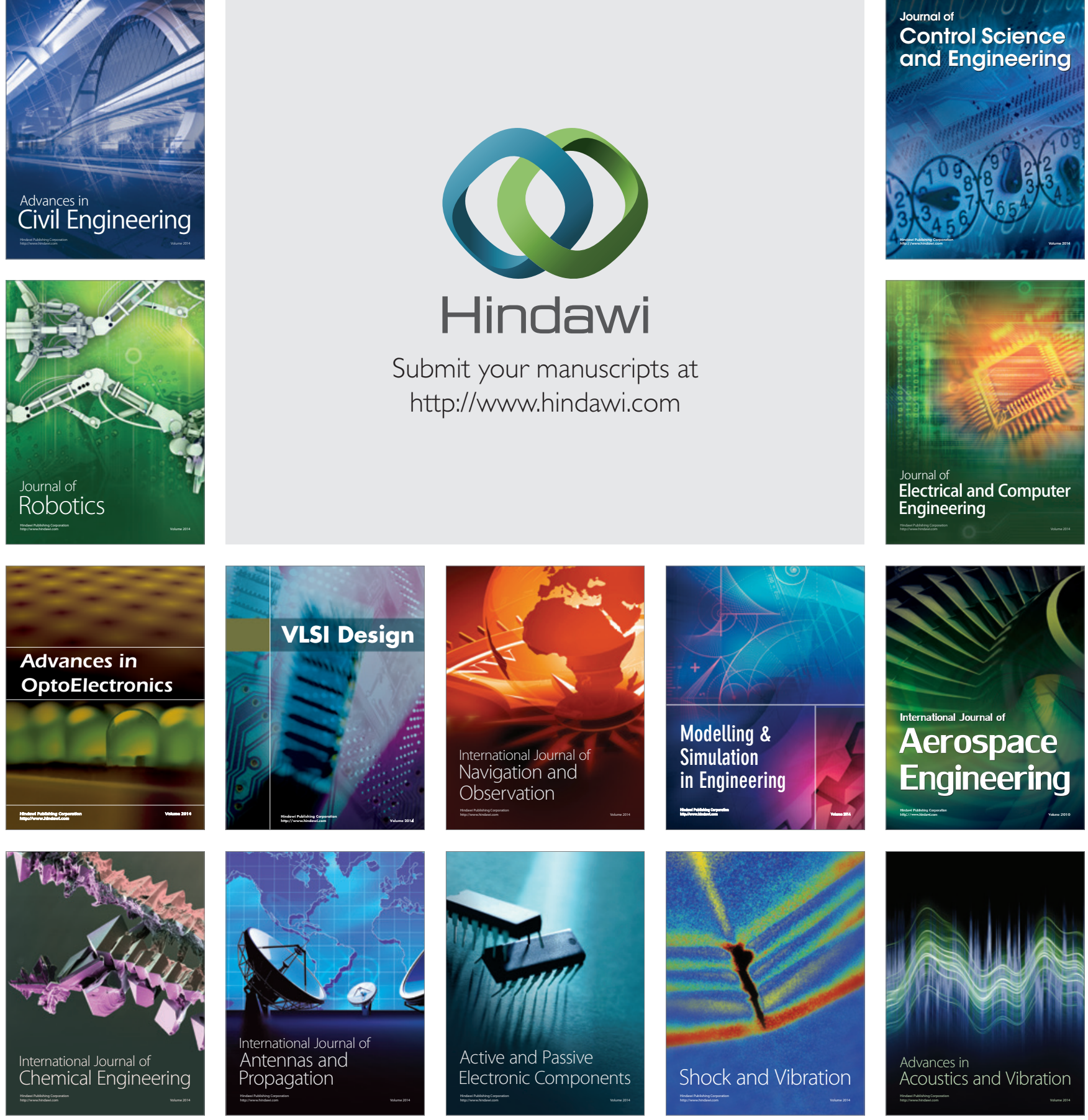